\title{
The glycine transporter 1 inhibitor SSR504734 enhances working memory performance in a continuous delayed alternation task in C57BL/6 mice
}

\author{
Journal Article \\ Author(s): \\ Singer, Philipp; Feldon, Joram; Yee, Benjamin K. \\ Publication date: \\ 2009 \\ Permanent link: \\ https://doi.org/10.3929/ethz-b-000009419
}

Rights / license:

In Copyright - Non-Commercial Use Permitted

Originally published in:

Psychopharmacology 202(1-3), https://doi.org/10.1007/s00213-008-1286-5 


\title{
The glycine transporter 1 inhibitor SSR504734 enhances working memory performance in a continuous delayed alternation task in C57BL/6 mice
}

\author{
Philipp Singer • Joram Feldon • Benjamin K. Yee
}

Received: 29 March 2008 / Accepted: 29 July 2008 / Published online: 29 August 2008

(C) Springer-Verlag 2008

\begin{abstract}
Rationale Inhibition of the glycine transporter 1 (GlyT1) activity increases extra-cellular glycine availability in the CNS. At glutamatergic synapses, increased binding to the glycine-B site located in the $N$-methyl-D-aspartate receptor (NMDAR) can enhance neurotransmission via NMDARs. Systemic treatment of 2-chloro- $N-[(S)$-phenyl [(2S)-piperidin2-yl] methyl]-3-trifluoromethyl benzamide, monohydrochloride (SSR504734), a selective GlyT1 inhibitor, is effective against social recognition impairment induced by neonatal phencyclidine treatment and enhances pre-pulse inhibition in a mouse strain (DBA/2) with intrinsic sensorimotor gating deficiency, suggesting that SSR504734 may be an effective cognitive enhancer.

Objective The objective of the study was to examine if SSR504734 exhibits a promnesic effect on working memory function in wild-type $\mathrm{C} 57 \mathrm{BL} / 6$ mice using an automatic continuous alternation task.

Materials and methods Hungry mice were trained to alternate their nose pokes between two food magazines across successive discrete trials in an operant chamber in order to obtain food reward. Correct choice on a given trial thus followed a non-matching or win-shift rule in relation to the preceding trial, with manipulation of the demand on memory retention, by varying the delay between successive trials.

Results Pre-treatment with SSR504734 (30 mg/kg, i.p.) improved choice accuracy when the delay from the previous trial was extended to $12-16 \mathrm{~s}$. Furthermore, a
\end{abstract}

P. Singer $\cdot$ J. Feldon $\cdot$ B. K. Yee $(\bowtie)$

Laboratory of Behavioural Neurobiology,

Swiss Federal Institute of Technology,

Schorenstrasse 16,

8603 Schwerzenbach, Switzerland

e-mail: byee@ethz.ch dose-response analysis $(3,10,30 \mathrm{mg} / \mathrm{kg}$ ) revealed a clear dose-dependent efficacy of the drug: $3 \mathrm{mg} / \mathrm{kg}$ was without effect, whilst $10 \mathrm{mg} / \mathrm{kg}$ led to an intermediate enhancement in performance.

Conclusion The present findings represent the first demonstration of the promnesic effects of SSR504734 under normal physiological conditions, lending further support to the suggestion of its potential as a cognitive enhancer.

Keywords Glycine transporter $1 \cdot$ Memory $\cdot$ Learning · NMDA receptor . Working memory

\section{Introduction}

The hypothesis that $N$-methyl-D-aspartate receptor (NMDAR) activation plays a central role in the neural plasticity underlying learning and memory (Morris 1989, Collingridge and Bliss 1995, Tang et al. 1999) has generated growing interest in agents that can enhance NMDAR activation as potential therapeutics against cognitive deficits caused by diseases and their potential use as cognitive enhancers in otherwise healthy individuals (Sahakian and Morein-Zamir, 2007). Because the simultaneous binding of both glutamate and glycine to NMDAR is mandatory for NMDAR ion channel opening (McBain and Mayer 1994), both the glutamate binding site located on the NMDAR NR2 subunit and the glycine binding site (glycine-B site) on the NR1 subunit constitute potential targets to enhance NMDAR activation. Due to a number of undesirable side effects associated with competitive NMDAR agonists acting at the glutamate site (Danysz and Parsons 1998), a current focus is on targeting the glycine-B site. It has long been suggested that the glycine-B sites are not saturated under physiological conditions (Danysz et al. 1987, 1989), and the subsequent 
confirmation of this hypothesis (Berger et al. 1998; Bergeron et al. 1998, Chen et al. 2003) has led to the suggestion that NMDAR activation can be facilitated by increasing glycine-B site occupancy. In support of this hypothesis, glycine-B site agonist (glycine and D-serine) as well as partial agonist (D-cycloserine) treatment can improve the therapeutic efficacy of conventional anti-psychotic medications against negative and cognitive symptoms of schizophrenia (Coyle and Tsai 2004, Heresco-Levy 2000) that are closely linked to NMDAR hypofunction (Olney and Farber 1995). Whilst there is no convincing evidence that glycine-B site agonists or partial agonists improve cognitive functions in healthy humans, this has however been demonstrated in laboratory animals in tests of associative learning, working memory, reversal learning, and familiarity judgement (Monahan et al. 1989, Pussinen and Sirviö 1999, Duffy et al. 2008, Nunnink et al. 2007, Zlomuzica et al. 2007).

One potential limit to the use of glycine or D-serine to potentiate NMDAR activation is that high systemic doses are required to significantly elevate their availability within the CNS because they are extensively metabolised and pass poorly through the blood-brain barrier (D'Souza et al. 2000). Furthermore, existing synthetic glycine-B site agonists have relatively low affinity in comparison to the endogenous agonists, glycine and D-serine (Milan 2002, Bräuner-Osborne et al. 2000). Indeed, it has been argued that the simple molecular structure of glycine in itself poses a limitation on the development of novel synthetic analogues (Milan 2002).

Alternatively, glycine-B site activation can be enhanced indirectly through pharmacological blockade of glycine transporter 1 (GlyT1). GlyT1 is a major reuptake regulator of glycine concentration at glutamatergic synapses (Aragon and Lopez-Corcuera 2005) and is co-localised with NMDARs (Attwell et al. 1993). Its normal physiological role and anatomical localisation indicate that pharmacological inhibition of GlyT1 would be a highly effective strategy to increase glycine-B site occupancy, overcoming the limitations of systemic treatment with glycine-B site agonists/partial agonists as described above. Amongst the growing number of GlyT1 inhibitors available, some have been shown to possess the pre-clinical profile of putative anti-psychotics and are currently undergoing clinical evaluation (Lechner 2006). However, there is until now only limited evidence for their potential pro-cognitive effects in non-pathological conditions. The most promising GlyT1 inhibitor in this regard is SSR504734, which has been shown to reverse the social recognition impairment in rats induced by neonatal phencyclidine treatment (Depoortère et al. 2005, Harich et al. 2007) and to enhance pre-pulse inhibition (PPI) in a mouse strain (DBA/2) with intrinsic sensorimotor gating deficiency (Depoortère et al. 2005). PPI is often taken as a measure of sensorimotor gating, which refers to a form of earlyattentional control in selective information processing, central to the prevention of sensory overload (Graham 1975, Swerdlow et al. 2000). Impaired PPI may be linked to other cognitive deficits, and, therefore, restoration of deficient PPI may alleviate such associated cognitive dysfunctions. There is also recent evidence suggesting that the PPI magnitude correlates with mnemonic performance in various learning tasks (Giakoumaki et al. 2006, Bitsios et al. 2006), suggesting that PPI enhancement may be a predictor of pro-cognitive properties. This lends further support to the hypothesis that SSR504734 may possess pro-cognitive potential.

The present study aimed to extend the behavioural assessment of SSR504734 in cognitive function (Depoortère et al. 2005) to working memory function in normal mice as a test of the drug's promnesic potential in non-pathological conditions. Here, working memory was assessed using an automated operant continuous delayed alternation procedure that readily allows the manipulation of delay intervals between successive trials, thus varying the retention demand on working memory function.

\section{Materials and methods}

\section{Subjects}

The subjects were 31 naïve adult male $\mathrm{C} 57 \mathrm{BL} / 6$ mice bred in the specific pathogen-free breeding facility (ETH Zurich Laboratory of Behavioural Neurobiology, Schwerzenbach, Switzerland). They were weaned at post-natal day 21 and caged in groups of unisex littermates until 10 weeks old, when they were transferred to single caging in Type II cages (Techniplast, Milan, Italy). The animals were maintained in a temperature- $\left(21^{\circ} \mathrm{C}\right)$ and humidity-controlled $(55 \%)$ vivarium under a 12:12 h reversed light-dark cycle (lights on at $1900 \mathrm{~h}$ ). Food and water were provided ad libitum until 1 week prior to behavioural training. Behavioural testing took place in the dark phase of the cycle and commenced when the animals were 12 weeks old. During the course of the experiment, the animals were maintained on a 23-h food deprivation regime, with their body weight reduced to not less than $85 \%$ of their ad lib weight. Water was available throughout.

The experimental manipulations and procedures described here had been previously approved by the Zurich Cantonal Veterinary Office. All procedures conformed fully to the ethical standards required by the Swiss Act and Ordinance on Animal Protection and the European Council Directives 86/609/EEC.

\section{Apparatus}

The experiments were conducted using four Habitest System (Coulbourn Instruments, Allentown, PA, USA) 
operant chambers (Model E10-10), each placed inside a ventilated and sound-attenuated chest. The internal dimensions of each chamber were $31 \times 25 \times 33 \mathrm{~cm}$, but an opaque Plexiglas partition wall was installed to reduce the available floor area to $15 \times 25 \mathrm{~cm}$. Illumination inside the chamber was provided by a house light $(2.8 \mathrm{~W})$ positioned on the panel wall $21 \mathrm{~cm}$ above the grid floor. Two food magazine trays were mounted on the panel wall, each equipped with a 1.4-W light source mounted inside. They were separately connected to two independent food pellet dispensers (Model H14-22M-20) using $20 \mathrm{mg}$ sucrose pellets (BioServ, Frenchtown, NJ, USA) as food reward. Nose poke responses (magazine entries) were detected by an infrared beam positioned at the entrance to each food magazine. The magazine trays were positioned on either side of a retractable lever mounted in the middle of the panel wall (Model H21-03M). A 2.8-W stimulus light was mounted $10 \mathrm{~cm}$ directly above the lever. Two additional transparent partitioning walls $(5 \mathrm{~cm}$ long and $6 \mathrm{~cm}$ apart) were mounted on either side of the retractable lever so that the animal was required to take a detour to move between the lever and either magazine tray.

\section{Drugs}

SSR504734 (2-chloro- $N$-[(S)-phenyl [(2S)-piperidin-2-yl] methyl]-3-trifluoromethyl benzamide, monohydrochloride) was donated by Sanofi-Aventis (Paris, France). The compound SSR504734 was suspended in distilled water containing 5\% Tween 80 (see Depoortère et al. 2005). Both SSR504734 and vehicle-control (5\% Tween) solutions were freshly prepared daily and injected via the intraperitoneal (i.p.) route $30 \mathrm{~min}$ before testing. The injected volume was $10 \mathrm{ml} / \mathrm{kg}$.

Acquisition of the delayed alternation task

\section{Introduction of food deprivation}

At the age of 10 weeks, the animals were singly housed and maintained on an ad lib food regime for 1 week prior to the commencement of the food deprivation. This was introduced gradually, with a progressive reduction of feeding time across 6 days $(12,6,4,2,1$, and $1 \mathrm{~h})$. Water was constantly available. At the same time, the animals were familiarised with the reward pellets in the home cage to minimise food neophobia when operant training began. Operant training commenced on the next day, and the animals were maintained with $1 \mathrm{~h}$ free access to food per day throughout the experimental period. The animals' body weight was monitored daily and was not permitted to fall below $85 \%$ of their ad lib weight.

\section{Magazine training}

The animals were first habituated to the operant chambers and allowed to consume food pellets freely from the food magazines. Each session began with ten food pellets already placed in each magazine tray. The house light and magazine lights were switched on throughout the $20 \mathrm{~min}$ of the session. Once all mice were reliably consuming the reward pellets, they were trained to earn reward by alternating their successive nose poke responses between the two magazines.

\section{Nose pokes training with signalled alternation}

Under the constant illumination of the house light, the session (once per day) began with the illumination of one of the two magazine trays. A nose poke into this magazine resulted in the delivery of one reward pellet. This magazine stayed illuminated for two additional seconds before being turned off, and at the same time, the alternative magazine was then illuminated awaiting for a nose poke response. Nose pokes directed at an unlit magazine tray did not yield any programmed consequence. The session ended with the cessation of the house light, either when the animal had made 16 correct nose poke responses or when $10 \mathrm{~min}$ had elapsed. When an animal had completed the 16 correct responses within the maximum time allowed on two consecutive days, it was trained to press a lever following each rewarded nose poke response in order to trigger the illumination of the next appropriate food magazine (see below).

\section{Lever press training with trial-discrete signalled nose pokes alternation}

With the house light on throughout, this training session (once per day) began with the presentation of the lever, which coincided with the illumination of the lever stimulus light directly above. One lever press resulted in the retraction of the lever, cessation of the lever stimulus light, and illumination of one of the two food magazines. Upon a nose poke to the illuminated magazine, one reward pellet was delivered to that magazine tray. The magazine light was then switched off after $2 \mathrm{~s}$, and the next trial commenced with the presentation of the lever signalled by the illumination of the lever stimulus light. Nose pokes into an unlit magazine tray would not yield any programmed consequence. The session was terminated with the cessation of the house light when either 16 trials had been completed or $10 \mathrm{~min}$ had elapsed. Delayed alternation commenced the day after the animals had completed the session within the maximum time on two consecutive days.

\section{Baseline delayed alternation training}

In the delayed alternation task, the animals were required to alternate successive nose pokes between the two food 
magazines to earn a reward. This essentially followed a "win-shift" rule, by which the correct response on a given trial was to shift away from the previous rewarded response. Choice accuracy thus depended on memory of the last rewarded response. The information relevant to the solution was trial dependent; hence, the task taxed one key aspect of working memory function (Honig 1978). The retention demand on working memory was manipulated here by varying the delays (i.e. the inter-trial intervals) between a (rewarded) response to the next choice.

The present delayed alternation task adopted a "two food magazines and one lever" configuration, which differs its design from the conventional trial-discrete non-matchingto-sample operant working memory task based on a "two levers and one food magazine" configuration (e.g. Dunnett 1993). However, this set-up was deliberately chosen because our intention to mimic the T-maze continuous delayed alternation procedure (e.g. see Marquis et al. 2007), whereby the animals were required to alternate their successive arm entries between the left and right choice stems on a T-maze to obtain food reward. The continuous nature of this task design was preserved in our operant procedure, such that the response on a give choice trial served effectively as the sample run of the following choice in accordance with the non-matching rule. This design has also been adopted previously in delayed non-matching-tosample tests (e.g. Aggleton et al. 1986, 1992, Yee and Rawlins 1994). Here, the use of a correction trial (see below) further ensured that correct choice behaviour would also be consistently guided by a win-shift strategy.

A schematic of the delayed alternation test procedures is provided in Fig. 1. With the house light on, each daily session began with an initial "forced" trial, in which a randomly selected magazine was illuminated. A nose poke into this magazine resulted in the delivery of a reward pellet. The magazine light remained on for two additional seconds, followed by the initiation of the inter-trial interval (ITI) preceding the first "choice" trial. A choice trial began with the presentation of the lever and the simultaneous illumination of the lever stimulus light, while both magazine lights remained off. A lever press resulted in the retraction of the lever, cessation of the lever light, and the illumination of both magazines. A correct nose poke response was followed by the immediate delivery of a reward pellet, cessation of the alternative magazine light, and the continual illumination of the chosen magazine light for $2 \mathrm{~s}$, followed then by the next ITI. On the other hand, an incorrect choice resulted immediately in the cessation of all magazine lights and a 5-s time-out period, during which the house light was switched off and the lever remained retracted. At the end of the time-out period, a forced trial using the previous correct magazine was administered as described above (also see Fig. 1). This was then followed

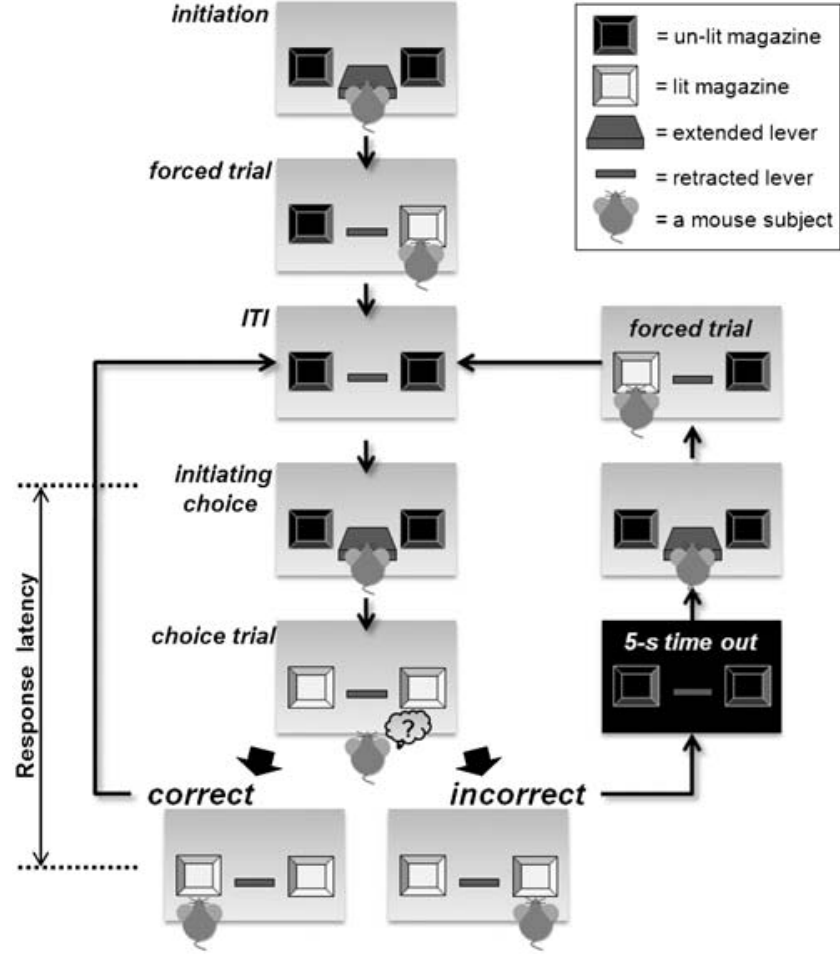

Fig. 1 Schematic illustration of the delayed alternation task. The session began with the presentation of the centrally located lever, the depression of which led to the start of a "forced trial", in which one of the two food magazines was illuminated waiting for a nose poke response. Upon detection of a nose poke into one of the two magazines, a food pellet was delivered into this magazine, and the first ITI delay was initiated. During the ITI period, the lever remained retracted and the magazine lights were off. At the end of the ITI, the lever was presented, and its depression was followed by its retraction and illumination of both magazines. Nose poke into the magazine alternative to the one that yielded a reward now resulted in the delivery of a reward (correct response). Nose poke into the magazine that previously yielded a reward (incorrect response) was followed by a 5-min "time-out" and then the presentation of a forced trial, in which only the correct magazine was illuminated. Upon a nose poke into this magazine, the programme resumed to the next appropriate ITI period, followed then by a choice trial. Response latency was defined as the time between lever press that triggered the magazine lights and the magazine entry (nose poke), which constituted to an explicit choice

by the appropriate ITI prior to the initiation of the next choice trial.

At the beginning of baseline training, all animals were trained using an ITI of $1 \mathrm{~s}$, with each session consisting of a maximum of 12 choice trials to be completed within a maximum of $10 \mathrm{~min}$. Animals that consistently failed to complete the 12 trials within the allotted time were excluded from further testing. Otherwise, when choice accuracy achieved $\geq 90 \%$ correct on two consecutive daily sessions, a variable ITI procedure was introduced. Within a session, the ITI varied between one of two possible delays counterbalanced amongst the 12 trials. Over the first 4 days, the ITI was either 1 or $4 \mathrm{~s}$. Over the next 4 days, the ITI was either 1 or $8 \mathrm{~s}$. This allowed us to confirm and to 
identify the sensitivity of the task to increasing ITI within sessions. A total of 17 mice reached this stage of training, and they were then given 2 days of rest before the next phase of testing with compound SSR504735.

Experiment 1: effects of SSR504734 on delayed alternation (1- vs 4- vs 8-s delays)

We started by evaluating the effect of systemic SSR504734 on performance in the delayed alternation task in two delayfunction experiments. We elected to focus first on the specific dose of $30 \mathrm{mg} / \mathrm{kg}$ i.p. because this was found to be behaviourally effective by Depoortère et al. (2005) and because it is also consistent with our impression obtained from initial pilot studies with this compound (unpublished data by P. Singer, J. Feldon and B.K. Yee).

In experiment 1, the delay function included three possible ITI delays: 1, 4 and $8 \mathrm{~s}$, inter-mixed across the 12 choice trials per session. On day 1 , all animals were given a vehicle injection 30 min before delayed alternation training to familiarise them with the injection procedure. The animals were subdivided into two groups-balanced with respect to their baseline performance (on day 1). Over the next 3 days, one group received daily pre-treatment of SSR504734 at $30 \mathrm{mg} / \mathrm{kg}(n=8)$ and the other of vehicle injection $(n=9)$. One SSR504734-treated subject was excluded because it failed to complete the 12 trials on the first two tests days. At the end of this experiment, the animals were given 2 days of rest before they were tested again in experiment 2. However, another mouse (from the vehicle group) died in this period, leaving a total of 15 mice available, before experiment 2 commenced.

Experiment 2: effects of SSR504734 on delayed alternation (1- vs 8 - vs 12- vs 16-s delays)

In experiment 2, the delay function included four possible ITI delays: $1,8,12$, and $16 \mathrm{~s}$, inter-mixed across the 16 choice trials per session. First, animals were given one session of pre-training, in which they all received a vehicle injection $30 \mathrm{~min}$ before the test (as in experiment 1). The animals $(n=15)$ were then subdivided into two new subgroups in order to counterbalance their previous drug experience in experiment 1. Namely, half of SSR504734treated animals in experiment 2 had received SSR504734 in experiment 1 and the other half vehicle in experiment 1 ; and half of vehicle-treated animals in experiment 2 had received SSR504734 in and the other half vehicle in experiment 1 . This allowed us to gauge the possible effects of previous experience with the drug. The two new groups (SSR504734: $n=7$, vehicle: $n=8$ ) received either daily pretreatment of SSR504734 $(30 \mathrm{mg} / \mathrm{kg}$, i.p. $)$ or vehicle solution as described above. Drug testing lasted for three consecutive days, after 1 day of training with vehicle injection in all animals (as described in "Experiment 1: Effects of SSR504734 on delayed alternation (1- vs 4- vs 8-s delays)"). After this, the animals were again given a break of 2 days prior to the next experiment.

Experiment 3: dose-response analysis of SSR504734's effect in delayed alternation

We then conducted a dose-response analysis of SSR504734 $(0,3,10$ and $30 \mathrm{mg} / \mathrm{kg})$ focussing on the minimal delay of $1 \mathrm{~s}$ and the extended delay of $12 \mathrm{~s}$. All subjects had completed experiments 1 and 2 before. On day 1, all animals received a vehicle injection as described before for a practice session. Over the following 4 days, a Latin square design was adopted to balance the order of drug doses across subjects $(n=15)$. Each daily session consisted of 12 choice trials, with half of the trials following a 1-s ITI delay and the other half a 12-s delay. A maximum time of 10 min per session was allowed. No further animals were excluded for not completing sessions.

\section{Dependent measures and statistical analysis}

Working memory performance was indexed by percent correct choices, at each delay condition on each day. In addition, the number of nose pokes recorded during the ITI delays on a given day was also calculated and separately analysed. All statistical analyses were carried out using SPSS $^{\circledR}$ for Windows ${ }^{\mathrm{TM}}$ (version 13) on a PC running the Windows XP (SP2) operating system. Parametric analyses of variance (ANOVA) were performed with the betweensubject factors, drug and previous drug experience, and the within-subjects factors, delays and days (in "Experiment 1: effects of SSR504734 on delayed alternation (1- vs 4- vs 8-s delays)" and "Experiment 2: effects of SSR504734 on delayed alternation (1- vs 8- vs 12- vs 16-s delays)"). In experiment 3 , the effect of drug dosage was investigated using a Latin square design, and the factor of drug doses was analysed as a within-subjects factor. Data interpretation was further assisted by Fisher's least significant difference (LSD) post hoc comparisons and the use of restricted ANOVAs for determining the locus of the significant effects. A critical level of $p<0.05$ was set for all statistical analyses.

\section{Results}

Delayed alternation baseline training

Baseline training on the continuous delayed alternation task began after the animals had successfully completed the pre- 
training of trial-discrete signalled nose poke alternation, in which no mnemonic demand was required. The baseline training on the continuous delayed alternation procedure consisted of three consecutive phases, lasting for 15, 4 and 4 days, respectively. In the first phase, all ITIs were $1 \mathrm{~s}$ in duration. As illustrated in Fig. 2A, choice accuracy indexed by percentage of correct trials per daily session was already above chance on day 1 . Afterwards, performance continued to improve steadily over days, achieving criterion performance by the 15th day. Repeated measures ANOVA of percent correct over the 15 days yielded a main effect of days $[F(14,238)=11.96, p<0.001 ; n=18)$.

In the second phase of baseline training, the ITIs varied between 1 and $4 \mathrm{~s}$. One animal failed repeatedly to complete the 12 trials within the time limit and was therefore excluded from the data analysis and from further testing. The total number of mice was thereby reduced to 17. As shown in Fig. 2B, increasing the ITI to $4 \mathrm{~s}$ did not result in any observable impact on choice accuracy in comparison with the minimal 1-s delay condition. A $2 \times 4$ (delays $\times$ days) repeated measures ANOVA of percent correct response failed to yield any significant effects.

In the third phase, the ITI within each daily session was either 1 or $8 \mathrm{~s}$. Choice accuracy was substantially reduced by the extension of ITI to $8 \mathrm{~s}$ in comparison with the high choice accuracy maintained at the 1-s delay condition (Fig. 2C). A $2 \times 4$ (delays $\times$ days) repeated measures ANOVA of percent correct response yielded a highly significant effect of delays $[F(1,17)=36.69, p<0.001]$. Neither the main effect of days nor its interaction with delays achieved statistical significance.

The results from the baseline phase indicated that the 4-s delay condition may be useful for assessing treatment expected to impair working memory function. On the other hand, delays of $8 \mathrm{~s}$ and above were sufficiently taxing for normal animals and would therefore be suitable for the detection of performance enhancing agents. The results of phases 2 and 3 here also verified the stability of performance at the minimal 1-s delay condition, even when it was inter-mixed with trials of a higher mnemonic demand as in phase 3. This design of varying delays within-session enjoys a potential advantage over a between-session design because the latter may lead to profound reduction of choice accuracy, such that the subjects might give up on the task.

Experiment 1: delay function of 1, 4 and $8 \mathrm{~s}$

One animal belonging to the SSR504734 condition repeatedly failed to complete the 12 trials within the time limit and was therefore excluded from the data analysis of experiment 1 and discontinued from further testing. The final number of subjects was therefore: SSR504734 $n=7$ and vehicle $n=9$. As shown in Fig. 3A, performance of both SSR504734and vehicle-treated animals was maintained at a high ( $90 \%)$ and comparable level at delays of 1 and $4 \mathrm{~s}$ across the 3 days of drug test. Choice accuracy expressed as mean percentage correct at different delays is depicted in Fig. 3A. A group difference emerged at the longest delay of $8 \mathrm{~s}$, with SSR504734-treated animals outperforming the vehicle controls. SSR504734 thus improved working memory performance in a delay-dependent manner, i.e. only when the demand on temporal retention was high.

These interpretations of the data were supported by a $2 \times$ $3 \times 3$ (drug $\times$ delays $\times$ days) ANOVA of percentage of correct across the 3 days of test, which yielded a significant main effect of delays $[F(2,28)=21.01, p<0.001]$ and its interaction with drug $[F(2,28)=7.39, p<0.005]$. Separate analyses

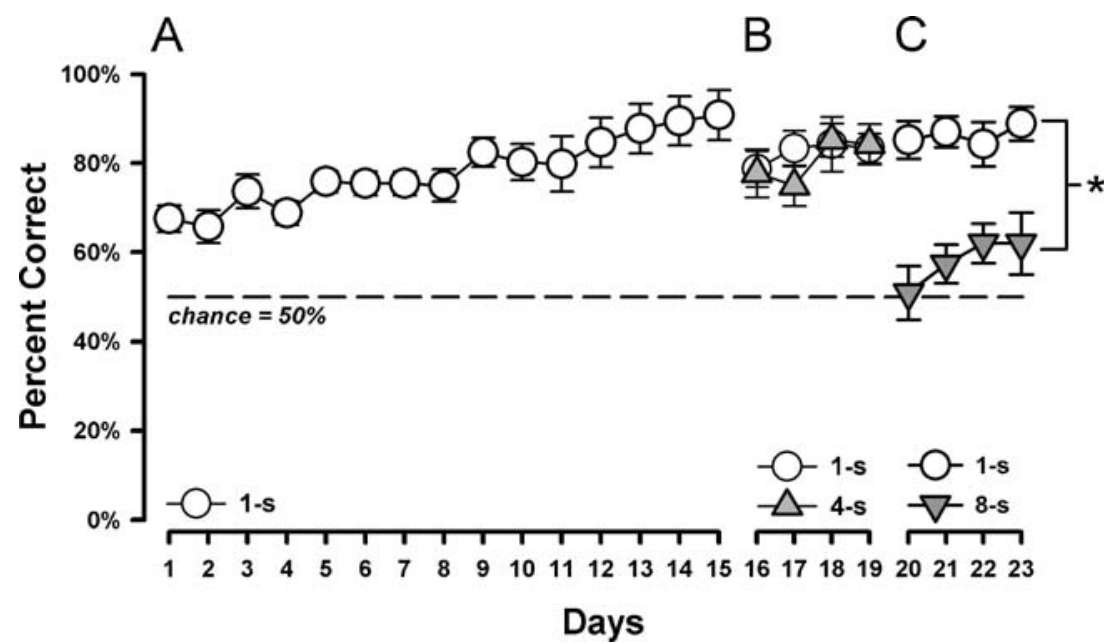

Fig. 2 Baseline delayed alternation training. (A) Acquisition of the delayed alternation task under the minimal (1-s ITI) delay condition. Performance was indexed by percent correct responses expressed as a function of daily sessions. $(B)$ Within-session comparison between trials following a 1-s ITI and those following a 4-s ITI indicated little evidence for an impact by this limited increase in retention demand. $(C)$ Withinsession comparison between trials following a 1-s ITI and those following a 4-s ITI yielded a significant main effect of delays $\left(^{*} p<\right.$ 0.005 ) as confirmed by a $2 \times 4$ (delays $\times$ days) repeated measures ANOVA of percent correct per day $(n=17)$. All values refer to mean \pm SEM 

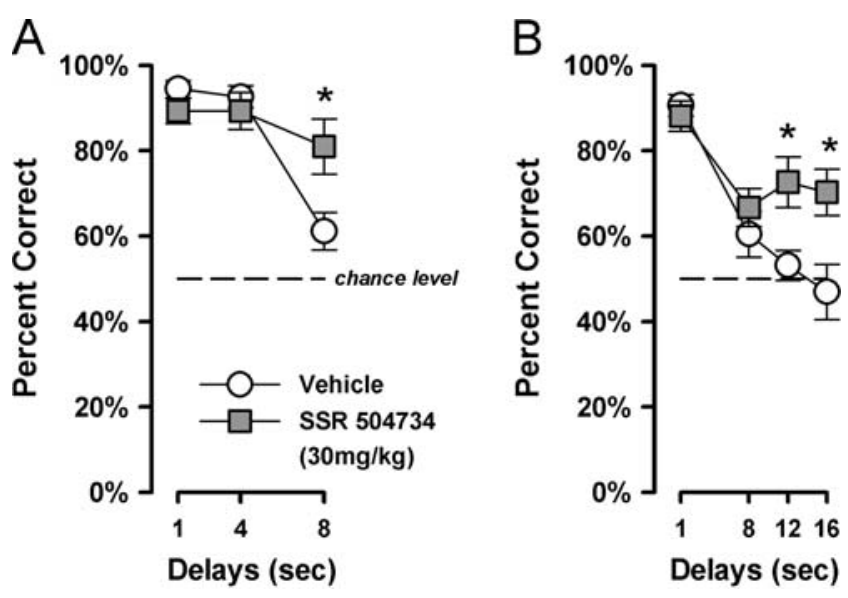

Fig. 3 Effects of systemic SSR504734 on the delayed alternation task. Two separate delay-function experiments (experiments 1 and 2, depicted in $\mathbf{A}$ and $\mathbf{B}$, respectively) were conducted to examine the effects of SSR504734 on delayed alternation performance. Animals used in experiment 2 were previously tested in experiment 1 . Drug experience was fully counterbalanced (see "Materials and methods"). Choice accuracy in each experiment was indexed by percent correct (average across three drug test days) and illustrated here as a function of ITI delays based on data obtained on three testing days. In both experiments, SSR504734 significantly improved choice accuracy in comparison to vehicle treatment at longer delays. Significant betweengroups difference at $* p<0.05$. Error bars refer to \pm SEM

restricted to each delay condition indicated that SSR504734 significantly enhanced choice accuracy only at 8 -s delay $[F(1,14)=6.88, p=0.02]$ but not at the two shorter delays. Furthermore, at the critical delay of 8 s when SSR504734 treatment exhibited a positive effect on performance, the controls were still able to maintain performance above chance ( $p<0.05$ based on one sample $t$ test). This suggested that the superior performance by the SSR504734 group could not be attributed to non-performance in the control group at this particular delay condition. The effects of the drug on performance did not appear to depend on days: neither the main effect of days nor any of its interactions achieved statistical significance. Indeed, analysis of data derived from day 1 only (when the SSR504734-treated mice were receiving the drug for the first time) were sufficient to yield a nearsignificant effect of drug by delays interaction $[F(2,28)=3.15$, $p=0.058]$ and a main effect of delays $[F(2,28)=5.60$, $p<0.05]$. When the comparison was restricted to the 8-s delay condition on day 1 , the drug effect again approached statistical significance $[F(1,14)=4.43, p=0.055]$, but did not do so at the shorter delay conditions $(F<1)$.

In addition, a separate analysis of the number of nose pokes recorded during all ITIs (when both magazine trays were not lit) was conducted. Such nose pokes were a potential source of retroactive interference that might affect working memory performance. A $2 \times 3$ (drug $\times$ days) ANOVA of this measure did not yield any significant effects. The frequency of nose pokes during ITIs was stable across days and comparable between treatment groups. The mean numbers of ITI nose pokes $( \pm$ SEM) per daily session were: $\operatorname{SSR} 504734=59.3 \pm 7.1$, vehicle control $=62.1 \pm 4.7$.

Experiment 2: delay function of $1,8,12$ and $16 \mathrm{~s}$

In the second delay-function experiment, the temporal demand of retention was increased by extending the delay up to $16 \mathrm{~s}$. Again, 3 days of drug test were conducted following 1 day of vehicle injection practice session in all subjects. The subjects' previous drug experience (in "Experiment 1: Effects of SSR504734 on delayed alternation (1- vs 4- vs 8-s delays)") was counterbalanced in the allocation of drug condition in experiment 2 . Thus in each drug condition here, there were subjects that had received the drug in experiment 1 and subjects that did not. Prior drug experience in experiment 1 was included as a between-subject factor in the analysis of the present data set, but this factor was excluded in the final analysis because it did not achieve statistical significance either as a main effect or interaction.

As depicted in Fig. 3B, SSR504734 again selectively enhanced choice accuracy at longer delays of 12 and $16 \mathrm{~s}$. The drug was, however, no longer effective at the 8-s delay condition (c.f. "Experiment 1: Effects of SSR504734 on delayed alternation (1- vs 4- vs 8-s delays)"), yet the general impression of a performance enhancing effect by SSR504734 was essentially replicated. Under the more extended delays of 12 and $16 \mathrm{~s}$, the vehicle group was no longer able to sustain above chance performance (based on one sample $t$ test), whereas the SSR504734 group maintained performance at about $70 \%$ correct.

These interpretations of the data were supported by a $2 \times$ $4 \times 3$ (drug $\times$ delays $\times$ days $)$ ANOVA of percent correct responses, which yielded a significant main effect of drug $[F(1,13)=8.50, p<0.02]$, delays $[F(3,39)=19.10, p<0.001]$ and their interaction $[F(3,39)=3.45, p<0.05]$. Again, there was no statistical evidence that performance varied significantly across days: Neither the main effect of days nor its interactions achieved statistical significance. Additional restricted analyses at each delay condition confirmed that SSR504734 treatment significantly enhanced choice accuracy when the delay was extended to $12 \mathrm{~s}[F(1,13)=8.48$, $p<0.02]$ and $16 \mathrm{~s}[F(1,13)=7.39, p<0.02]$.

Analysis of the number of nose pokes made during ITI delays per daily session by a $2 \times 3$ (drug $\times$ days) ANOVA again yielded no significant effects. The mean numbers of nose pokes $( \pm$ SEM) per daily session were: SSR504734= $106.9 \pm 10.8$, vehicle control $=102.4 \pm 9.8$.

Experiment 3: dose-response analysis

Finally, we conducted a dose-response analysis across doses: $0,3,10$ and $30 \mathrm{mg} / \mathrm{kg}$ by focussing on the minimal 
delay of $1 \mathrm{~s}$ and the extended delay of $12 \mathrm{~s}$ to ascertain whether a positive drug effect might also be identified at lower doses. The animals from experiments 1 and $2(n=15)$ were employed here in a Latin square design to best counterbalance across drug doses in a within-subject manner.

SSR504734 dose dependently enhanced choice accuracy, but this effect was most clearly demonstrated in the 12-s delay condition (Fig. 4). The efficacy of the highest dose $(30 \mathrm{mg} / \mathrm{kg}$ ) was again confirmed here, with the $10-\mathrm{mg} / \mathrm{kg}$ dose producing an intermediate level of enhancement. These contrasted with the lack of effect at the lowest dose of $3 \mathrm{mg} / \mathrm{kg}$.

A $4 \times 2$ (drug doses $\times$ delays) repeated measures ANOVA of choice accuracy yielded a significant main effect of drug doses $[F(3,42)=5.76, p<0.005]$ and delays $[F(1,14)=$ $241.43, p<0.001]$. Despite the tendency apparent in Fig. 4, the interaction term failed to attain statistical significance. However, separate analyses restricted to each delay condition revealed a significant effect of drug doses only in the 12-s delay condition $[F(3,42)=3.93, p<0.05]$. Planned contrast analysis of this significant effect indicated that only the highest dose $(30 \mathrm{mg} / \mathrm{kg})$ significantly enhanced performance in comparison with vehicle treatment $(p<0.05)$.

Separate one-way repeated measures ANOVAs of nose poke frequency during ITI delays yielded no significant effect of drug doses. The mean numbers of nose pokes $( \pm$ SEM) per daily session were: vehicle-control $=60.2 \pm 6.0,3 \mathrm{mg} / \mathrm{kg}=$ $63.1 \pm 6.1,10 \mathrm{mg} / \mathrm{kg}=50.0 \pm 6.1,30 \mathrm{mg} / \mathrm{kg}=65.6 \pm 7.1$.

As a further test of the robustness of our results here, which were obtained using a Latin square design and where

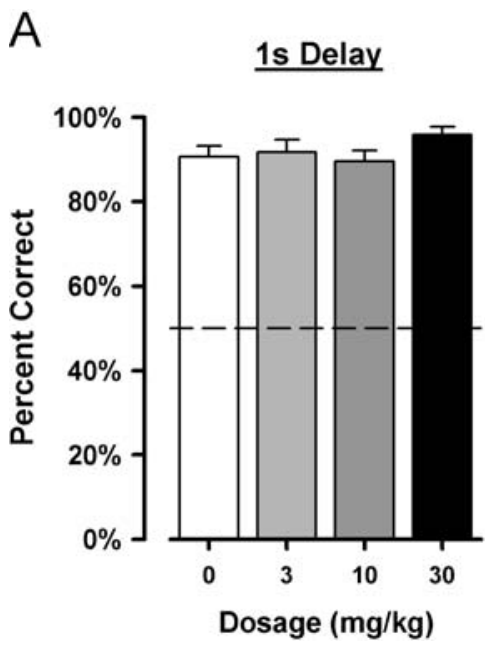

Fig. 4 Dose-response analysis of SSR504734 on delayed alternation performance. The efficacy to enhance delayed alternation performance by SSR 504734 at three doses $(3,10$ and $30 \mathrm{mg} / \mathrm{kg}$ ) in comparison with vehicle treatment was assessed for two specific ITI delays: at the minimal 1-s delay condition (A) and the extended 12-s delay condition (B). A Latin square design was employed to counterbalance drug experiences across four drug tests days. Data presented are based on the factor dose was evaluated in a within-subject manner, we performed an additional analysis restricted to the first test day, in which the same factor was analysed as a between-subject factor. In spite of the anticipated reduction in statistical power, this nonetheless would allow an assessment of whether the overall pattern of results described above was already apparent on the first day. To this end, a $4 \times 2$ (drug doses $\times$ delays) ANOVA was performed on this restricted data set, which revealed a pattern of results almost identical to the overall analysis across the four test days. The analysis yielded a significant effect of delay $F(1,11)=86.3, p<0.001]$ and of dose $[F(3,11)=3.83, p<0.05]$. Post hoc LSD comparisons further confirmed the efficacy of SSR504734 to enhance performance at the highest dose $(30 \mathrm{mg} / \mathrm{kg})$ in comparison with vehicle treatment $(p=0.006)$. In the minimal 1 -s delay condition, choice accuracy across increasing dose $(0,3,10$ and $30 \mathrm{mg} / \mathrm{kg}$ ) was: $83.3 \pm 3.9 \%, 95.8 \pm 3.9 \%, 91.7 \pm 3.9 \%$ and $94.4 \pm 4.5 \%$, respectively. In the 12 -s delay condition, the corresponding percent correct scores were: $41.7 \pm 7.3 \%$, $50.0 \pm 7.3 \%, 54.2 \pm 7.3 \%$ and $77.8 \pm 8.4 \%$, respectively.

\section{Discussion}

The present study sought to evaluate the promnesic potential of SSR504734 on working memory functions using an operant delayed alternation paradigm. Across three experiments, the results revealed that SSR504734 reliably enhanced working memory performance at a dose of $30 \mathrm{mg} / \mathrm{kg}$. The robustness of this particular dose demon-
$\mathrm{B}$

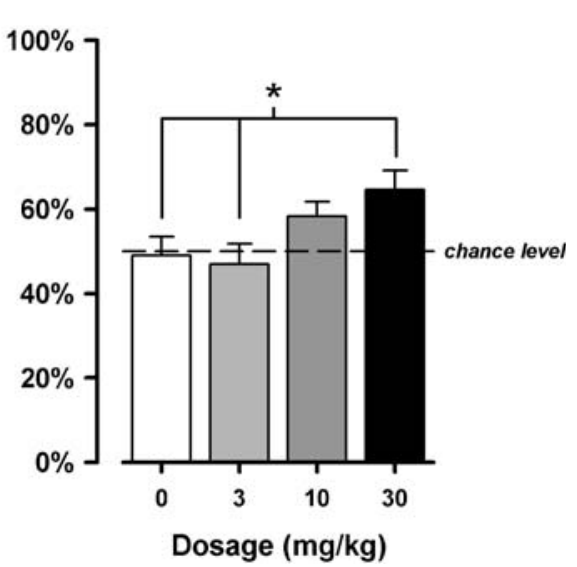

4 days of drug test. A clear dose-dependent effect was observed at the delay condition of 12 s: SSR504734 dose-dependently enhanced choice accuracy (B). Whilst the dose of $3 \mathrm{mg} / \mathrm{kg}$ lacked any clear effect, the highest dose employed here $(30 \mathrm{mg} / \mathrm{kg})$ was clearly effective $\left({ }^{*} p<0.05\right.$ in comparison to vehicle condition and $3 \mathrm{mg} / \mathrm{kg}$ ), with the middle dose $(10 \mathrm{mg} / \mathrm{kg})$ producing an intermediate effect. Error bars refer to mean \pm SEM 
strated here is in agreement with the report by Depoortère et al. (2005) who also showed that the dose of $30 \mathrm{mg} / \mathrm{kg}$ was consistently active across a number of behavioural paradigms relevant for schizophrenia-related cognitive deficits. In contrast, the lowest dose of $3 \mathrm{mg} / \mathrm{kg}$ was essentially devoid of any efficacy in the present task.

The promnesic effect of SSR504734 probably stems from the acute effect of the drug, although in each experiment, animals received multiple injections of the drug across daily sessions. The overall pattern of results in experiment 1 was evident when animals were first administered the drug on the first day of the experiment. The outcome of experiment 2 did not depend on whether the subjects were naïve to SSR504734 before the experiment. In experiment 3 , dose was varied over 4 days, and a response was clear. In addition, the overall pattern of results of experiment 3 was clearly evident on the first day of test. Thus, the robustness of the dose-response analysis was supported in that it did not depend on the uniqueness of the Latin square design.

Our results therefore implied that systemic treatment of SSR504734 had very limited long-term effect on the present memory task. This may represent an advantage from the perspective of a cognitive enhancing agent for the non-clinical population, but could also be a disadvantage as a therapeutic agent against chronic and persistent cognitive deficiency.

The positive effect of SSR504734 on choice accuracy expressed itself in a delay-dependent manner. This suggests that the drug did not indiscriminately elevate performance but was most effective when the delay condition was sufficiently taxing. We manipulated task difficulty primarily by varying the task's retention demand. In experiment 1 , the drug was only effective at 8 -s delays when performance of the controls began to deteriorate drastically, while remaining above the level of performance expected by chance alone. In experiment 2, although the drug appeared ineffective under the same delay conditions, its ability to improve performance was clearly evident at extended delays of 12 and $16 \mathrm{~s}$ and at a range at which the control failed to exceed the level of performance expected by chance alone. We have no explanation for the drug's lack of efficacy in experiment 2 at the 8-s delay condition, which contrasted with its demonstrated effect in experiment 1 . However, we can exclude the possibility that repeated dosing was the cause because the impact of prior drug exposure in experiment 1 did not affect the outcome of experiment 2 . We can also exclude the introduction of the 12- and 16-s delays in experiment 2 as a likely reason because the results obtained on the first test day (when the novelty of these test conditions was maximal) conformed to the outcome at 8 -s delays in experiment 1 . Thus, previous experience of experiment 1 probably is important. Alternatively, the 8-s delay condition was not functionally identical in the two experiments because its presentation was intermixed with different delay conditions. Nonetheless, this does not detract from our general conclusion that SSR504734 affects working memory function under varying retention demands.

From the end of the baseline training onwards, the animals had maintained a consistently high level of choice accuracy at performance at the minimal delay condition in all three experiments. It suggests that the animals had maintained the motivation to perform the task throughout the session in spite of increasing task difficulty imposed by the inclusion of more extended delays. This further strengthens our conclusion that SSR504734 can enhance mnemonic capacity with respect to memory retention and retrieval. It would be highly relevant and interesting to assess if the drug also possesses such potential when task difficulty is achieved by other task parameters, for example by varying the levels of interference.

One critical determinant of working memory function is the ability to overcome or resolve interference (Jonides and Nee 2006), and the present working memory task is no exception. Indeed, the levels of interference might be considerable. Every trial involved the same two choices (or discriminanda), namely the two magazine trays, which were identical in appearance except for their location in the operant chamber. The repeated use of a single pair of discriminanda in delayed matching-to-sample task (another working memory test) is considerably more difficult in comparison with the use of trial-unique discriminanda (e.g. Yee and Rawlins 1998). It is because memories acquired from preceding trials potentially interfere with the retrieval of the relevant information on a given trial. This source of (proactive) interference is, however, expected to reduce as the delay interval between trials is lengthened.

Furthermore, the animals made additional nose pokes into the inactive magazine trays (i.e. when the magazine lights were off) during the retention intervals. These responses represented another potential source of (retroactive) interference, making accurate recall of the relevant past information (location of the previous nose poke that generated reward) more difficult. It is not possible to prevent such responses in the present task design unless switchable magazine doors are installed. An alternative to overcome this interference is the "two levers and one magazine" configuration where the discriminanda (i.e. the levers) can be retracted during the inter-trial intervals. In contrast to proactive interference resulting from preceding trials, retroactive interference is expected to increase as the delay interval between trials widens.

Our data indicated that such retroactive interference was largely similar for both drug and vehicle conditions; thus, the possibility that SSR504734 enhanced performance here solely by reducing the occurrence of interfering inter-trial 
nose pokes could be excluded. This assertion, however, does not repudiate the possibility that SSR504734 may act by reducing the functional impact of retroactive interference on working memory function. For example, it may enhance the perceptual distinctiveness or discriminability between memory traces, thereby facilitating their selective retrieval. If so, SSR504734 may be generally effective in facilitating cognitive flexibility. This interesting possibility would certainly require additional tests in which interference is explicitly manipulated (e.g. Yee and Rawlins 1998, Sanderson et al. 2007).

The demonstrated delay-dependent effect of SSR504734 readily suggests that the drug has a promnesic impact on the animal's working memory capacity. It enhances the ability to retain and retrieve information across the intertrial intervals in order to respond correctly on the next trial. This characteristic of trial dependency is the hallmark of working memory according to the relevant theories by Honig (1978) and Olton (1979). The alternative win-shift strategy would also be expected to require recall of trialdependent memory because throughout the sessions, both magazine trays would be associated with rewards. In addition, the task can be solved based on relative familiarity (or recency) judgement because accurate solution can be achieved by always selecting the relatively less familiar magazine tray (Rawlins and Tsaltas 1983). This particular strategy would be most prone to the accumulation of interference and would predict that trials at the beginning of the session are easier than those at the end. We attempted to address this possibility by comparing performance between the two halves within a session using our current data sets. No significant difference in performance was however evident between the two halves of a test session (data not shown), although a consistent trend of lower accuracy in the last half (by approximately $5 \%$ points) relative to the first half was noted in all three experiments. This trend was similarly observed regardless of drug conditions, suggesting that the drug did not enhance performance primarily through a reduction in within-session proactive interference.

One potential problem associated with sequential alternation task (especially in an automated set-up) is the possible adoption of a motor mediating strategy (Olton et al. 1979), which may allow a solution that is essentially free from any working memory demand. Here, this possibility had been reduced by requiring the subjects to press a centrally located lever to activate the availability of a choice (illumination of both magazine lights), thus ensuring that the mouse would always be in the same location when the choice was presented. Furthermore, the installation of partition walls between the lever and magazines also reduced the use of simple motor pattern as mediating strategy, although this possibility can never be completely eliminated (see Stanhope et al. 1995). Most critically, however, the use of mediating behaviour is expected to assist performance at short rather than at long delays (Rawlins and Tsaltas 1983). Given that SSR504734 was primarily effective at longer delays, the mnemonic conclusion of this outcome is less likely to be weakened by the interpretative problems associated with the potential use of mediating strategy in such tasks.

Another relevant performance measure, as suggested by an anonymous reviewer, is the animals' response speed or latency to make a decision in the context of task difficulty as a function increasing delays. In our task, this corresponds to the time between the lever press that triggered the magazine lights and the nose poke that constituted the choice selection (see Fig. 1). We elected to focus on experiment 2 because it covered the widest spectrum of delays (Fig. 5). A distinction between correct and incorrect response was made, but there were insufficient data to allow such a distinction at 1-s delays because of the high level of performance in this condition. This analysis revealed that response latency for correct response was significantly higher than for incorrect response across delays of $8-16 \mathrm{~s}$ [see Fig. 5B; $F(1,13)=12.70, p>0.005$ ]. Response latency for correct responses showed an inverted $\mathrm{U}$-shaped function against increasing delays. This led to the emergence of a significant main effect of delays $[F(3,39)=$ $3.55, p<0.05]$ in a repeated measure ANOVA of the latency of correct responses across the four delay conditions, which was solely attributed to the quadratic component $[F(1,13)=$ 15.92, $p<0.005$; see Fig. 5A). Taken together, response latency may therefore reflect effortful retrieval, which was more demanding with increasing delays, but the animals eventually shifted away from such effortful recall when the delay was too high. In this respect, the non-significant protraction of response latency by SSR504734 may be relevant to the psychological impact of the drug on cognitive functioning in general, even though this tendency in itself may not fully account for the significant delaydependent enhancement of choice accuracy by SSR504734.

The present demonstration of a promnesic effect following GlyT1 inhibition is of critical importance because until now, clinical evidence on the pro-cognitive potential of GlyT1 inhibition is limited to the endogenous GlyT1 inhibitor sarcosine in the treatment of schizophrenia (Tsai et al. 2004, Lane et al. 2005). Moreover, although adjunct glycine therapy was found to improve negative as well as cognitive symptoms in schizophrenia patients (HerescoLevy et al. 1999, 2004, Javitt et al. 2001), studies in healthy volunteers had questioned the ability of acute high-dose glycine treatment to improve cognitive function in nonpathological conditions. Recently, Palmer et al. (2008) failed to obtain any evidence of cognitive enhancement in healthy subjects following treatment, with high doses of glycine $(0.8 \mathrm{~g} / \mathrm{kg}$, per os) in measures of working memory, 

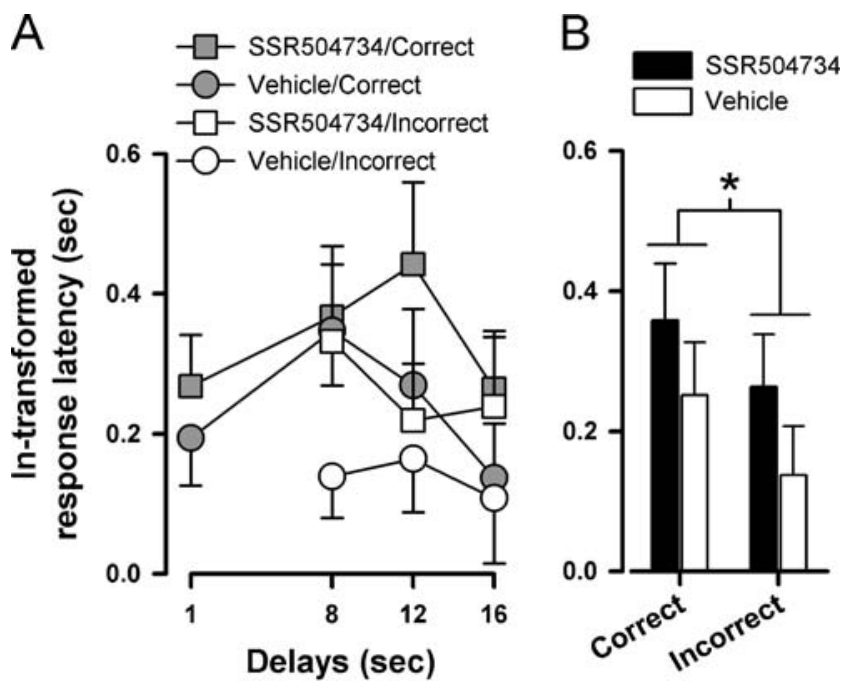

Fig. 5 Response latency as a function of delay and response in experiment 2. A Response latency (in seconds followed by lntransformation) as a function of delays in experiment 2 is represented by separate curves, according to drug treatment conditions and whether the response was correct or incorrect. In the 1-s delay condition, four mice did not make any error and therefore representative group means for incorrect response latency cannot be calculated. Error bars refer to \pm SEM. Analysis of correct responses across all four delay conditions by a $2 \times 4$ (drug $\times$ delays) ANOVA yielded a main effect of delays $[F(3,39)=3.55, p<0.05]$, which was associative with a significant effect exclusively in the quadratic trend $[F(1,13)=15.92$, $p<0.005$ ] according to an orthogonal contrast analysis, supporting the presence of an inverted U-shaped effect. To examine the difference in response latency between correct and incorrect response, the analysis was restricted to the three higher delay conditions by a $2 \times 2 \times 3$ (drug $\times$ response type $\times$ delays) ANOVA. This yielded a main effect of delays $[F(2,26)=4.52, p<0.05]$ and response type $[F(1,13)=12.70, p>0.005]$, while the main effect of drug and its interaction terms did not achieve statistical significance. B This depicts the mean response latency averaged across the delays conditions of 8,12 and $16 \mathrm{~s}$, illustrating the significant difference in response latency between correct and incorrect responses $\left({ }^{*} p<0.05\right)$ Error bars refer to \pm SEM

declarative memory, attention and perceptual processing. Interpretation is further complicated by reports that a single oral dose of glycine $(0.8 \mathrm{~g} / \mathrm{kg}$, per os $)$ can produce a negative impact on cognition as demonstrated in the test of mismatch negativity (Leung et al. 2008), in which schizophrenia patients also exhibit a deficit (e.g. Umbricht and Krljes 2005). Given that acute glycine administration at 0.1 to $0.2 \mathrm{mg} / \mathrm{kg}$ via the intraperitoneal route is highly effective in substantially elevating plasma and CSF levels of glycine within $2 \mathrm{~h}$ after injection (D'Souza et al. 2000), the oral dose of $0.8 \mathrm{mg} / \mathrm{kg}$ described above is considerably high in spite of the difference in the route of administration.

Why may high concentrations of glycine fail to enhance and even impair cognition? First, excessive elevations of glycine above the saturation threshold of glycine-B site can result in NMDAR internalisation and may thereby lead to a negative impact on overall NMDAR functions at the network levels. This can result from either endogenous glycine treatment (Nong et al. 2003) or GlyT1 blockade (Martina et al. 2004). Secondly, elevated glycine levels could also potentiate NMDARs located on inhibitory $\gamma$ aminobutyric acid interneurons (Javitt et al. 1993), which in turn would dampen overall excitatory neurotransmission at the network levels. Thirdly, neuronal inhibition can also result from the action of excessive glycine on low-affinity inhibitory glycine receptors on pyramidal neurons (Laube et al. 2002, Betz and Laube 2006, Rajendra et al. 1997). These possible physiological responses to global elevation of glycine concentration may imply that a moderate increase in the glycine levels is more favourable (Singer et al. 2007a, b).

Interestingly, Palmer et al. (2008) suggested that the lack of a pro-cognitive effect following acute glycine treatment may be due to individual variability in endogenous glycine concentration. Given that the optimal elevation of glycine for improving cognitive functions may differ considerably between subjects, individual titration would be necessary to show a consistent effect. The ability to adjust for the optimal glycine concentration is further hindered by the poor blood-brain barrier passage of glycine, which necessitates massive dosing: A typical dose of $0.8 \mathrm{~g} / \mathrm{kg}$ implies a daily intake of glycine exceeding $50 \mathrm{~g}$ for an adult human (Heresco-Levy et al. 1996, 1999, Javitt et al. 2001). The potential side effects under such conditions must be seriously considered. Besides the obvious respiratory problems associated with exposure to high doses of glycine, morphological changes in the cerebellum (e.g. a decrease in density of astocytic fibres) have been reported in rats following a 2-week glycine-rich diet (Shoham et al. 1999).

In view of the above limitations associated with direct glycine intake, blockade of GlyT1 represents a superior pharmacological approach to achieving selective potentiation of NMDAR activation aimed at enhancing cognitive functions. First of all, GlyT1 inhibitors are expected to readily pass through the blood-brain barrier (e.g. Molander et al. 2007) and therefore do not require exceptionally high doses to reach their targets. Secondly, given the close anatomical proximity between GlyT1 and NMDARs (Cubelos et al. 2005a, b), GlyT1 inhibition is ideally suited to achieve elevation of the functionally most relevant local concentration of glycine - that is in the vicinity of the NMDAR's glycine-B site. Hence, this would maximise the functional impact in terms of its desired action on NMDAR function, without inducing excessive changes to the global concentration of glycine in the brain, which, as explained above, could be counterproductive to the desired aim to enhance NMDAR activities.

There is considerable evidence to suggest that effective working memory function depends on both hippocampus and pre-frontal cortex. However, the sensitivity of the present task to hippocampal or pre-frontal cortex damage 
has not been validated in mice. We have thus far validated its sensitivity to systemic scopolamine treatment retrospectively, using the same animals examined here: A clear dosedependent effect of scopolamine was observed (Fig. 6), with significant impairment seen at $1 \mathrm{mg} / \mathrm{kg}$ (i.p.), and the effect appeared to be independent of delays (c.f., Dunnett et al. 1990). The pivotal contribution of NMDAR activity and glutamatergic transmission in the hippocampus to working memory has been suggested by Bannerman and colleagues (e.g. McHugh et al. 2008; Bannerman et al. 2006, 2008). SSR50473 has been shown to potentiate NMDAR-mediated excitatory post-synaptic currents in rat hippocampal slice preparations (Depoortère et al. 2005),

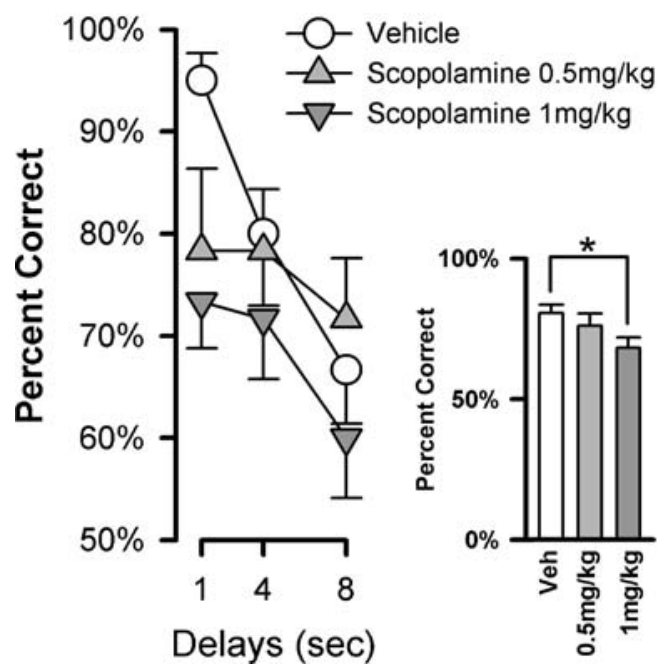

Fig. 6 Dose-dependent sensitivity of the delayed alternation test to systemic scopolamine. A dose-response analysis of scopolamine $(0,0.5$ and $1 \mathrm{mg} / \mathrm{kg}$ i.p. $)$ was conducted on the same animals that had completed the three SSR experiments reported here $(n=15)$. The animals were given 2 weeks for recovery after experiment 3 , before commencement of the scopolamine experiment. The experiment lasted for 5 days. On day 1 , all animals received 1 day of practice training on the 1- to 4- to 8-s delay function. On day 2 , a similar practice session took place with all animals receiving a saline $(0.9 \% \mathrm{NaCl})$ vehicle pretreatment. The effect of scopolamine was evaluated over the next 3 days. A Latin square design was employed to balance individual drug experience. The higher dose $(1 \mathrm{mg} / \mathrm{kg})$ clearly reduced choice accuracy as compared to the control, which was seen across all delays conditions. Choice accuracy following treatment at the lower dose $(0.5 \mathrm{mg} / \mathrm{kg})$ was intermediate between control and the higher dose, indicating that scopolamine disrupted performance in a dose-dependent manner. This gave rise to a significant main effect of dose $[F(2,28)=$ $3.50, p<0.05]$ and of delays $[F(2,28)=6.56, p<0.01]$ based on a $3 \times 3$ (dose $\times$ delays) ANOVA of percent correct. The impairment produced by $1 \mathrm{mg} / \mathrm{kg}$ scopolamine relative to vehicle treatment was confirmed by supplementary planned contrast analysis $[F(1,14)=9.88, p<0.01]$, but the deficit produced by $0.5 \mathrm{mg} / \mathrm{kg}$ scopolamine did not reach statistical significance $(p>0.3)$. The impairment produced by $1 \mathrm{mg} / \mathrm{kg}$ scopolamine did not appear to be delay dependent, given the absence of a significant dose $\times$ delays interaction. ${ }^{*} p<0.05$ based on planned contrast analysis applied to the within-subject factor of dose. Error bars refer to \pm SEM and this is consistent with the suggestion that enhanced NMDAR activation in the hippocampus had contributed to the working memory enhancement seen in the present study. Concerning the contribution of pre-frontal cortex to working memory function, the primary focus is on dopaminergic activity, especially via dopamine D1 receptors (for a review, see Brach 2004; Mehta and Riedel 2006), which may at first glance seem unrelated to the pharmacology of SSR50473. However, consistent with the relevance of prefrontal dopamine activity to working memory function, SSR50473 has been reported to increase the extra-cellular dopamine levels in pre-frontal cortex in vivo (Depoortère et al. 2005) and to facilitate dopamine release in the nucleus accumbens evoked by electrical stimulation of the basolateral amygdala (Leonetti et al. 2006). It is therefore possible that the more direct impact of SSR504734 on NMDAR-mediated glutamatergic neurotransmission within limbic cortices (e.g. hippocampus as well as amygdala) can subsequently lead to the potentiation of dopaminergic activity in pre-frontal cortex. Such concerted alternations in multiple brain regions following systemic SSR504734 administration may be responsible for the enhancement in working memory function demonstrated here. Further dissections of the relative contributions between different brain structures would necessitate additional experiments involving local intracerebral drug infusion.

\section{Conclusions}

The present report demonstrated, for the first time, that the enhancement of glycine availability in brain by inhibition of its uptake via the GlyT1 transporter can lead to a clear mnemonic improvement in non-pathological conditions. In particular, it adds to previous reports of SSR504734's efficacy to restore the social recognition deficits in rats induced by neonatal exposures to phencyclidine and to alleviate the sensorimotor gating deficit intrinsic to the DBA/2 mouse strain (Depoortère et al. 2005, Harich et al. 2007). The current novel finding thus substantiates the potential use of GlyT1 inhibitors, and specifically SSR504734, as adjunctive medication in the treatment of cognitive and negative schizophrenia symptoms (Kinney et al. 2003; Kinney and Sur 2005) and further extends its potential application in other domains of cognitive well-being (Lechner 2006) as highlighted by recent studies of GlyT1 knockout mice (Yee et al. 2006; Singer et al. 2007a, b).

Acknowledgements The present study was supported by the Swiss Federal Institute of Technology (ETH) Zurich and the NCCR Neural Plasticity \& Repair (funded by the Swiss National Science Foundation). The authors are also grateful to Dr. Bernard Scatton (Sanofi-Aventis, Paris, France) for his support for the present study and in providing the compound SSR504734. The authors also thank Peter Schmid for his 
excellent technical support, and the animal husbandry staff at the Laboratory of Behavioural Neurobiology, for their maintenance of the subjects used in the experiments.

Disclosure The experimental manipulations and procedures described here had been previously approved by the Swiss Cantonal Veterinary Office and conform to the ethical standards required by the Swiss Act and Ordinance on Animal Protection and the European Council Directives $86 / 609 /$ EEC. No conflicts of interest are to be declared by any of the authors of the present article.

\section{References}

Aggleton JP, Hunt PR, Rawlins JNP (1986) The effects of hippocampal lesions upon spatial and non-spatial tests of working memory. Behav Brain Res 19:133-146

Aggleton JP, Keith AB, Rawlins JN, Hunt PR, Sahgal A (1992) Removal of the hippocampus and transection of the fornix produce comparable deficits on delayed non-matching to position by rats. Behav Brain Res 52:61-71

Aragon C, Lopez-Corcuera B (2005) Glycine transporters: crucial roles of pharmacological interest revealed by gene deletion. Trends Pharmacol Sci 26:283-286

Attwell D, Barbour B, Szatkowski M (1993) Nonvesicular release of neurotransmitter. Neuron 11:401-407

Bannerman DM, Rawlins JN, Good MA (2006) The drugs don't workor do they? Pharmacological and transgenic studies of the contribution of NMDA and GluR-A-containing AMPA receptors to hippocampal-dependent memory. Psychopharmacology (Berl) 188:552-566

Bannerman DM, Niewoehner B, Lyon L, Romberg C, Schmitt WB, Taylor A, Sanderson DJ, Cottam J, Sprengel R, Seeburg PH, Köhr G, Rawlins JNP (2008) NMDA receptor subunit NR2A is required for rapidly acquired spatial working memory but not incremental spatial reference memory. J Neurosci 28:3623-630

Barch DM (2004) Pharmacological manipulation of human working memory. Psychopharmacology (Berl) 174:126-135

Berger AJ, Dieudonné S, Ascher P (1998) Glycine uptake governs glycine site occupancy at NMDA receptors of excitatory synapses. J Neurophysiol 80:3336-3340

Bergeron R, Meyer TM, Coyle JT, Greene RW (1998) Modulation of N-methyl-D-aspartate receptor function by glycine transport. Proc Natl Acad Sci USA 95:15730-15734

Betz H, Laube B (2006) Glycine receptors: recent insights into their structural organization and functional diversity. $\mathrm{J}$ Neurochem 97:1600-1610

Bitsios P, Giakoumaki SG, Theou K, Frangou S (2006) Increased prepulse inhibition of the acoustic startle response is associated with better strategy formation and execution times in healthy males. Neuropsychologia 44:2494-2499

Bräuner-Osborne H, Egenbjerg J, Nielsen E, Madsen U, KrogsgaardLarsen P (2000) Ligands for glutamate receptors: design and therapeutic prospects. J Med Chem 43:2609-2645

Chen L, Muhlhauser M, Yang CR (2003) Glycine tranporter-1 blockade potentiates NMDA-mediated responses in rat prefrontal cortical neurons in vitro and in vivo. J Neurophysiol 89:691-703

Collingridge GL, Bliss TVP (1995) Memories of NMDA receptors and LTP. Trends Neurosci 18:54-56

Coyle JT, Tsai G (2004) NMDA receptor function, neuroplasticity, and the pathophysiology of schizophrenia. Int Rev Neurobiol 59:491-515

Cubelos B, Gimenez C, Zafra F (2005a) Localization of the GLYT1 glycine transporter at glutamatergic synapses in the rat brain. Cereb Cortex 15:448-459
Cubelos B, Gonzales-Gonzales IM, Gimenez C, Zafra F (2005b) The scaffolding protein PSD-95 interacts with the glycine transporter GLYT1 and impairs its internalization. J Neurochem 95:10471058

D'Souza DC, Gil R, Cassello K, Morrissey K, Abi-Saab D, White J, Sturwold R, Bennett A, Karper LP, Zuzarte E (2000) IV glycine and oral D-cycloserine effects on plasma and CSF amino acids in healthy humans. Biol Psychiat 47:450-462

Danysz W, Parsons AC (1998) Glycine and N-methyl-D-aspartate receptors: physiological significance and possible therapeutic applications. Pharmacol Rev 50:597-664

Danysz W, Wroblewski JT, Brooker G, Costa E (1987) Modulation of excitatory amino acid transmission by phencyclidine and glycine in the rat cerebellum in vivo. Soc Neurosci Abstr 13:383

Danysz W, Wroblewski JT, Brooker G, Costa E (1989) Modulation of glutamate receptors by phencyclidine and glycine in the rat cerebellum: cGMP increase in vivo. Brain Res 479:270-276

Depoortère R, Estenne-Bouhtou G, Coste A, Lanneau C (2005) Neurochemical, electrophysiological and pharmalogical profiles of the selective inhibitor of the glycine transporter-1 SSR504734, a potential new type of antipsychotic. Neuropsychopharmacology 30:1963-1985

Dunnett SB (1993) Operant delayed matching and non-matching to position in rats. In: Sahgal A (ed) Behavioural neuroscience. A practical approach, vol. 1.. IRL, Oxford, pp 123-136

Dunnett SB, Wareham AT, Morres EM (1990) Cholinergic blockade in prefrontal cortex and hippocampus disrupts short-term memory in rats. Neuroreport 1:61-64

Duffy S, Labrie V, Roder JC (2008) D-serine augments NMDANR2B receptor-dependent hippocampal long-term depression and spatial reversal learning. Neuropsychopharmacology 33: 1004-1018

Giakoumaki SG, Bitsios P, Frangou S (2006) The level of prepulse inhibition in healthy individuals may index cortical modulation of early information processing. Brain Res 1078:168-170

Graham FK (1975) The more or less startling effects of weak prestimulation. Psychophysiology 12:238-248

Harich S, Gross G, Bespalov A (2007) Stimulation of the metabotropic glutamate $2 / 3$ receptor attenuates social novelty discrimination deficits induced by neonatal phencyclidine treatment. Psychopharmacology (Berl) 192:511-519

Heresco-Levy U (2000) N-Methyl-D-aspartate (NMDA) receptorbased treatment approaches in schizophrenia: the first decade. Int J Neuropsychopharmacol 3:243-258

Heresco-Levy U, Javitt DC, Ermilov M, Mor, del C, Horowitz A, Kelly D (1996) Double-blind, placebo-controlled, crossover trial of glycine adjuvant therapy for treatment-resistant schizophrenia. Br J Psychiat 169:610-617

Heresco-Levy U, Javitt DC, Ermilov M, Mordel C, Silipo G, Lichtenstein M (1999) Efficacy of high-dose glycine in the treatment of enduring negative symptoms of schizophrenia. Arch Gen Psychiat 56:29-36

Heresco-Levy U, Ermilov M, Lichtenberg P, Bar G, Javitt DC (2004) High-dose glycine added to olanzapine and risperidone for the treatment of schizophrenia. Biol Psychiat 55:165-171

Honig WK (1978) Studies of working memory in the pigeon. In: Hulse SH, Fowler H, Honig WK (eds) Cognitive processes in animal behavior. Erlbaum, Hillsdale, pp 211-248

Javitt DC, Doneshka P, Zylberman I, Ritter W, Vaughan HG Jr (1993) Impairment of early cortical processing in schizophrenia: an eventrelated potential confirmation study. Biol Psychiat 33:513-519

Javitt DC, Silipo G, Cienfuegos A, Shelley AM, Bark N, Park M, Lindenmayer JP, Suckow R, Zukin SR (2001) Adjunctive high dose glycine in the treatment of schizophrenia. Int $\mathrm{J}$ Neuropsychopharmacol 4:385-391

Jonides J, Nee DE (2006) Brain mechanisms of proactive interference in working memory. Neuroscience 139:181-193 
Kinney GG, Sur C (2005) Glycine site modulators and transporter-1 inhibitors as novel therapeutic targets for the treatment of schizophrenia. Curr Neuropharmacol 3:35-43

Kinney GG, Sur C, Burno M, Mallorga PJ, Williams JB, Figueroa DJ, Wittmann M, Lemaire W, Conn PJ (2003) The glycine transporter type 1 inhibitor N-[3-(4_-fluorophenyl)-3-(4_-phenylphenoxy) propyl]-sarcosine potentiates NMDA receptor-mediated responses in vivo and produces an antipsychotic profile in rodent behavior. $\mathrm{J}$ Neurosci 23:7586-7591

Lane HY, Chang YC, Liu YC, Chiu CC, Tsai GE (2005) Sarcosine or D-serine add-on treatment for acute exacerbation of schizophrenia: a randomized, double-blind, placebo-controlled study. Arch Gen Psychiat 62:1196-1204

Laube B, Maksay G, Schemm R, Betz H (2002) Modulation of glycine receptor function: a novel approach for therapeutic intervention at inhibitory synapses? Trends Pharmacol Sci 23:519-527

Lechner SM (2006) Glutamate-based therapeutic approaches: inhibitors of glycine transport. Curr Opin Pharmacol 6:75-81

Leonetti M, Desvignes C, Bougault I, Souilhac J, Oury-Donat F, Steinberg R (2006) 2-Chloro-N-[(S)-phenyl [(2S)-piperidin-2-yl] methyl]-3-trifluoromethyl benzamide, monohydrochloride, an inhibitor of the glycine transporter type 1, increases evoked-dopamine release in the rat nucleus accumbens in vivo via an enhanced glutamatergic neurotransmission. Neuroscience 137:555-564

Leung S, Croft RJ, O’Neill BV, Nathan PJ (2008) Acute high-dose glycine attenuates mismatch negativity (MMN) in healthy human controls. Psychopharmacology (Berl) 196:451-460

Marquis JP, Audet MC, Doré FY, Goulet S (2007) Delayed alteration performance following subchronic phencyclidine administration in rats depends on task parameters. Prog Neuropsychopharmacol Biol Psychiatry 31:1108-1112

Martina M, Gorfinkel Y, Halman S, Lowe JA, Periyalwar P, Schmidt CJ, Bergeron R (2004) Glycine transporter type 1 blockade changes NMDA receptor-mediated responses and LTP in hippocampal CA1 pyramidal cells by altering extracellular glycine levels. J Physiol 557:489-500

McBain CJ, Mayer ML (1994) N-Methyl-D-aspartic acid receptor structure and function. Physiol Rev 74:723-760

McHugh SB, Niewoehner B, Rawlins JNP, Bannerman DM (2008) Dorsal hippocampal N-methyl-D-aspartate receptors underlie spatial working memory performance during non-matching to place testing on the T-maze. Behav Brain Res 186:41-47

Mehta MA, Riedel WJ (2006) Dopaminergic enhancement of cognitive function. Current Pharmaceutical Design 12:2487-2500

Milan MJ (2002) N-Methyl-D-aspartate receptor-coupled glycine-B receptors in the pathogenesis and treatment of schizophrenia: a critical review. Curr Drug Targets CNS Neurol Disord 1:191-213

Molander A, Lidö HH, Löf E, Eriscon M, Söderpalm B (2007) The glycine reuptake inhibitor Org 25935 decreases ethanol intake and preference in mal wistar rats. Alcohol 42:11-18

Monahan JB, Handelmann GE, Hood WF, Cordi AA (1989) DCycloserine, a positive modulator of the N-methyl-D-aspartate receptor, enhances performance of learning tasks in rats. Pharmacol Biochem Behav 34:649-653

Morris RG (1989) Synaptic plasticity and learning: selective impairment of learning rats and blockade of long-term potentiation in vivo by the N-methyl-D-aspartate receptor antagonist AP5. J Neurosci 19:3040-3057

Nong Y, Huang YQ, Ju Wa, Kalia LV, Ahmadian G, Wang YT, Salter MW (2003) Glycine binding primes NMDA receptor internalization. Nature 422:302-307

Nunnink M, Davenport RA, Ortega B, Houpt TA (2007) D-Cycloserine enhances conditioned taste aversion learning in rats. Pharmacol Biochem Behav 87:321-330

Olney JW, Farber NB (1995) Glutamate receptor dysfunction and schizophrenia. Arch Gen Psychiat 52:998-1007
Olton DS (1979) Mazes, maps, and memory. Am Psychol 34:583-596

Olton DS, Becker JT, Handelmann GE (1979) Hippocampus, space and memory. Behav Brain Sci 2:313-365

Palmer C, Ellis KA, O'Neill BV, Croft RJ, Leung S, Oliver C, Wesnes KA, Nathan PJ (2008) The cognitive effects of modulating the glycine site of the NMDA receptor with high-dose glycine in healthy controls. Hum Psychopharmacol 23:151-159

Pussinen R, Sirviö P (1999) Effects of D-cycloserine, a positive modulator of N-methyl-D-aspartate receptors, and ST 587, a putative alpha-1 adrenergic agonist, individually and in combination, on the non-delayed and delayed foraging behaviour of rats assessed in the radial arm. J Psychopharmacol 13:171179

Rajendra S, Lynch JW, Schofield PR (1997) The glycine receptor. Pharmacol Ther 73:121-146

Rawlins JNP, Tsaltas E (1983) The hippocampus, time and working memory. Behav Brain Res 10:233-262

Sahakian B, Morein-Zamir S (2007) Professor's little helper. Nature 450:1157-1159

Sanderson DJ, Gray A, Simon A, Taylor AM, Deacon RM, Seeburg PH, Sprengel R, Good MA, Rawlins JN, Bannerman DM (2007) Deletion of glutamate receptor-A (GluR-A) AMPA receptor sunbunits impairs one-trial spatial memory. Behav Neurosci 121:559-569

Shoham S, Javitt DC, Heresco-Levy U (1999) High dose glycine nutrition affects glial cell morphology in rat hippocampus and cerebellum. Int J Neuropsychopharmacol 2:35-40

Singer P, Boison D, Möhler H, Feldon J, Yee BK (2007a) Enhanced recognition memory following glycine transporter 1 deletion in forebrain neurons. Behav Neurosci 121:815-25

Singer P, Feldon J, Yee BK (2007b) Interference of glycine transporter 1: modulation of cognitive functions via activation of glycine-B site of the NMDA receptor. CNS Agents Med Chem 7:259-268

Stanhope KJ, McLenachan AP, Dourish CT (1995) Dissociation between cognitive and motor/motivational deficits in the delayed matching to position test: effects of scopolamine, 8$\mathrm{OH}-\mathrm{DPAT}$ and EAA antagonists. Psychopharmacology (Berl) 122:268-280

Swerdlow NR, Braff DL, Geyer MA (2000) Animal models of deficient sensorimotor gating: what we know, what we think we know, and what we hope to know soon. Behav Pharmacol 11:185-204

Tang YP, Shimizu E, Dube GR, Rampon C, Kerchner GA, Zhuo M, Liu G, Tsien JZ (1999) Genetic enhancement of learning and memory in mice. Nature 401:63-69

Tsai G, Lane HY, Yang P, Chong MY, Lange N (2004) Glycine transporter I inhibitor, N-methylglycine (sarcosine), added to antipsychotics for the treatment of schizophrenia. Biol Psychiat 55:452-456

Umbricht D, Krljes S (2005) Mismatch negativity in schizophrenia: a meta-analysis. Schizophr Res 76:1-23

Yee BK, Rawlins JNP (1994) The effects of hippocampal formation ablation or fimbria-fornix section on performance of a nonspatial radial arm maze task by rats. J Neurosci 14:3766-3774

Yee BK, Rawlins JNP (1998) A comparison between the effects of medial septal lesions and entorhinal cortex lesions on performance of nonspatial working memory tasks and reversal learning. Behav Brain Res 94:281-300

Yee BK, Balic E, Singer P, Schwerdel C, Grampp T, Gabernet L, Knuesel I, Benke D, Feldon J, Mohler H, Boison D (2006) Disruption of glycine transporter 1 restricted to forebrain neurons is associated with a procognitive and antipsychotic phenotypic profile. J Neurosci 26:3169-3181

Zlomuzica A, De Souza Silva MA, Huston JP, Dere E (2007) NMDA receptor modulation by D-cycloserine promotes episodic-like memory in mice. Psychopharmacology (Berl) 193:503-509 